\title{
A Time-Kill Assay Study on the Synergistic Bactericidal Activity of Pomegranate Rind Extract and Zn (II) against Methicillin-Resistant Staphylococcus aureus (MRSA), Staphylococcus epidermidis, Escherichia coli, and Pseudomonas aeruginosa
}

\author{
Amal Alrashidi ${ }^{1}$, Mohammed Jafar ${ }^{1}$, Niamh Higgins ${ }^{1}$, Ciara Mulligan ${ }^{1}$, Carmine Varricchio ${ }^{1}$ (D), \\ Ryan Moseley $^{2}{ }^{(D}$, Vildan Celiksoy ${ }^{1,2} \mathbb{D}$, David M. J. Houston ${ }^{1}$ and Charles M. Heard ${ }^{1, * \mathbb{D}}$ \\ 1 School of Pharmacy and Pharmaceutical Sciences, Cardiff University, Cardiff CF10 3NB, UK; \\ alrashidia@cardiff.ac.uk (A.A.); jafarm@cardiff.ac.uk (M.J.); higginsn1@cardiff.ac.uk (N.H.); \\ mulligancf@cardiff.ac.uk (C.M.); varricchioc@cardiff.ac.uk (C.V.); celiksoyv@cardiff.ac.uk (V.C.); \\ houstondm@cardiff.ac.uk (D.M.J.H.) \\ 2 Oral and Biomedical Sciences, School of Dentistry, Cardiff University, Cardiff CF14 4XY, UK; \\ moseleyr@cardiff.ac.uk \\ updates \\ Citation: Alrashidi, A.; Jafar, M.;
} Higgins, N.; Mulligan, C.; Varricchio, C.; Moseley, R.; Celiksoy, V.; Houston, D.M.J.; Heard, C.M. A Time-Kill Assay Study on the Synergistic Bactericidal Activity of Pomegranate Rind Extract and Zn (II) against Methicillin-Resistant Staphylococcus aureus (MRSA), Staphylococcus epidermidis, Escherichia coli, and Pseudomonas aeruginosa. Biomolecules 2021, 11, 1889. https://doi.org/ 10.3390/biom11121889

Academic Editors: Eleonora Pagnotta, Luisa Ugolini and Giovanni Cilia

Received: 1 November 2021

Accepted: 13 December 2021

Published: 16 December 2021

Publisher's Note: MDPI stays neutral with regard to jurisdictional claims in published maps and institutional affiliations.

Copyright: (c) 2021 by the authors. Licensee MDPI, Basel, Switzerland. This article is an open access article distributed under the terms and conditions of the Creative Commons Attribution (CC BY) license (https:// creativecommons.org/licenses/by/ $4.0 /)$.

\begin{abstract}
There is a need for new antimicrobial systems due to increased global resistance to current antimicrobials. Pomegranate rind extract (PRE) and Zn (II) ions both possess a level of antimicrobial activity and work has previously shown that PRE/Zn (II) in combination possesses synergistic activity against Herpes simplex virus and Micrococcus luteus. Here, we determined whether such synergistic activity extended to other, more pathogenic, bacteria. Reference strains of methicillinresistant Staphylococcus aureus (MRSA), Staphylococcus epidermidis, Escherichia coli, and Pseudomonas aeruginosa were cultured and subjected to challenge by PRE, Zn (II), or PRE + Zn (II), in time-kill assays. Data were obtained independently by two researchers using different PRE preparations. Statistically significant synergistic activity for PRE + Zn (II) was shown for all four bacterial strains tested compared to untreated controls, although the extent of efficacy and timescales varied. $\mathrm{Zn}$ (II) exerted activity and at $1 \mathrm{~h}$, it was not possible to distinguish with PRE + Zn (II) combination treatment in all cases. PRE alone showed low activity against all four bacteria. Reproducible synergistic bactericidal activity involving PRE and Zn (II) has been confirmed. Potential mechanisms are discussed. The development of a therapeutic system that possesses demonstrable antimicrobial activity is supported which lends itself particularly to topical delivery applications, for example MRSA infections.
\end{abstract}

Keywords: PRE; zinc; synergistic activity; time-kill assay; Staphylococcus aureus (MRSA); Staphylococcus epidermidis; Escherichia coli; Pseudomonas aeruginosa

\section{Introduction}

Antimicrobial resistance is a natural phenomenon, occurring as a consequence of gradual changes in bacterial gene expression to facilitate the development of resistance against certain antibiotic modalities. The current global antibiotic resistance crisis has been particularly attributed to the overuse and misuse of these therapeutics, in addition to other factors-such as inappropriate antibiotic prescribing, extensive agricultural use, and the lack of new antibiotic drug development-have all contributed to the dramatic emergence of modern drug-resistant pathogens [1,2]. With the recent dramatic increases in resistance to existing antimicrobial agents, certain infections have become extremely difficult to treat, with generally stricter regulations being applied to antibiotic administration. For example, 
methicillin-resistant Staphylococcus aureus (MRSA) infection is caused by Staphylococcus aureus that has developed resistance to many of the antibiotics used to treat such infections, which typically occur in people who have been in hospitals and are difficult to treat. Therefore, there is an urgent need to economically develop more novel agents and approaches to treat microbial infections. This is an important consideration, as the accessibility of affordable new medicines to middle-to-low-income countries has been recognised as a priority by World Health Organization (WHO), where the use of traditional and complementary medicines is emphasised, as the costs of developing new single chemical entities would be prohibitive [3]. Furthermore, there is a recognised predilection of the populous for medicines that are of natural origin.

The pomegranate, the fruit of Punica granatum L., has a longstanding history as a folklore medicine and in the treatment of various bacterial infections, which has been generally supported having been wide number of studies in recent times [4]. Its anti-microbial properties have been largely attributed to the polyphenolic contents, of which the hydrolysable ellagitannins are the dominant class [5]. Such phytochemicals are concentrated in the fruit exocarp (or 'rind') although other extracts of other parts of the fruit-such as the arils-also possess anti-microbial/bactericidal effects [6]. Al-Zoreky (2002) demonstrated that pomegranate peel exhibited bactericidal activity against Listeria monocytogenes, Staphylococcus aureus, Escherichia coli, Salmonella enteritidis, and Yersinia enterocolitica [7]. Similarly, Nozohour et al. (2018) demonstrated the activity of pomegranate rind extract (PRE) against methicillin-resistant Staphylococcus aureus (MRSA) and Pseudomonas aeruginosa [8]. Studies have shown that punicalagin (Figure 1) and its metabolites such as ellagic acid, punicalin and the gut metabolite urolithin A-possess a range of antioxidant, anti-inflammatory, and antimicrobial properties $[9,10]$.

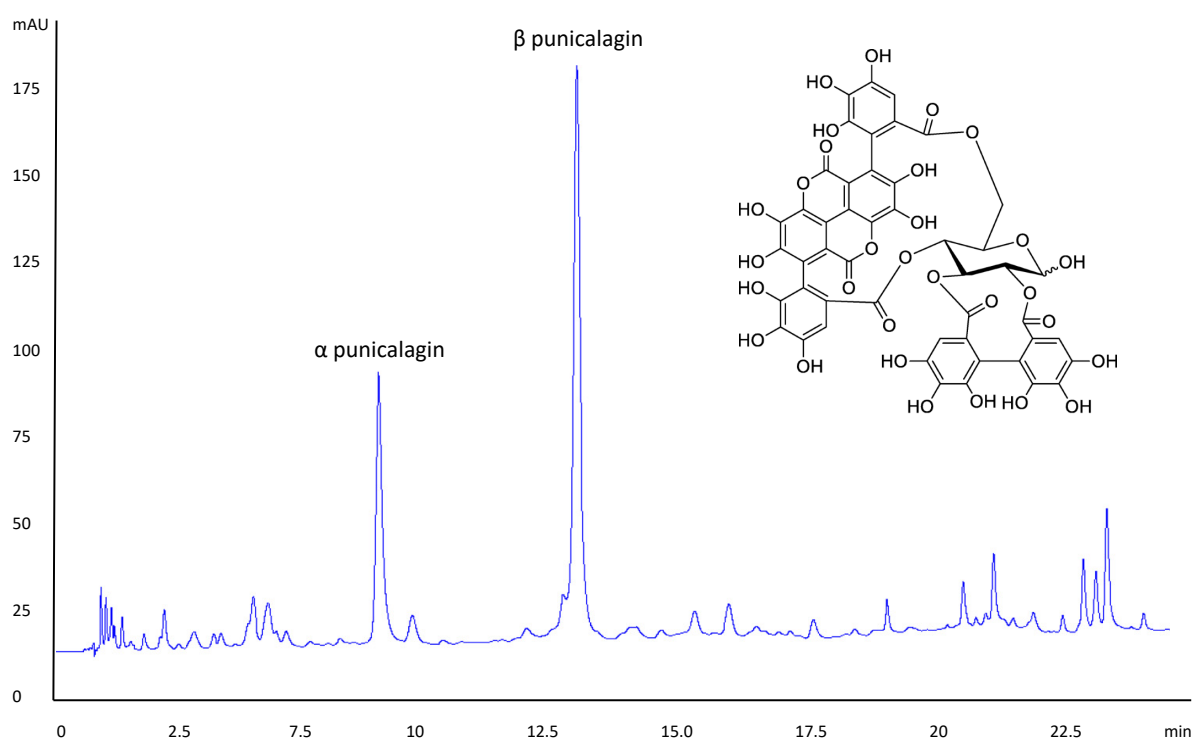

Figure 1. HPLC chromatogram showing PRE elution and highlighting the peaks due to the major ellagitannin punicalagin ( $\alpha$ and $\beta$ anomers), which showed characteristic 1:2 $\alpha / \beta$ ratio; inset: chemical structure of punicalagin. The level of punicalagin, which is known to be the main active component, was $21.7 \%$ (or $1 \mathrm{mg}$ punicalagin contained within $4.61 \mathrm{mg}$ of PRE). NB $\alpha$ and $\beta$ punicalagin spontaneously exist in this ratio. The full spectrum of components of PRE is described elsewhere.

Although it is widely recognised that punicalagin is largely responsible for the bioactivity of PRE, there is a rationale for developing holistic pomegranate extracts as antimicrobials. It has been reported that $>120$ different phytochemicals are found in pomegranate extracts, and the significance of combination therapy in combating resistance mechanisms is of increasing interest [11-13]. Multidrug resistance can arise through several different resistance genes, each providing resistance to a particular antibiotic, or a single resistance mechanism giving resistance to more than one antibiotic [14]. For example, extended 
spectrum $\beta$ lactamase (ESBL)-producing Gram-negative bacteria like E. coli and Klebsiella pneumoniae. ESBLs are enzymes that inhibit the activity of many clinically important antibiotics. Thus, infections with bacteria expressing ESBLs are hard to treat and are becoming increasingly common [15].

In another facet of exploring combination therapies, natural products have been combined with other substances to achieve enhanced or synergistic action, including as anti-microbials [16]. In particular, the enhancement of PRE activity has been explored with the co-application of transition metal ions, including Fe (II) and Cu (II) $[17,18]$, and notably $\mathrm{Zn}$ (II), with significant synergistic (potentiated) activities having been found against the Herpes simplex virus (HSV-1, HSV-2 and acyclovir-resistant HSV-2) [19] and bacteria, such as Micrococcus luteus [20].

In an effort to further explore the broad-spectrum nature of the PRE/Zn (II) combinational therapy, the current work aimed to determine if the same general conditions that displayed synergistic bactericidal activity reported previously, particularly against the bacterium $M$. luteus, would also be observed against a panel of other bacteria of a more pathogenic nature. In this paper, we examined the time-kill effects of PRE, Zn (II) and PRE/Zn (II) combination against the Gram-positive bacteria, MRSA, and Staphylococcus epidermidis and the Gram-negative bacteria, Escherichia coli, and Pseudomonas aeruginosa.

\section{Materials and Methods}

\subsection{Materials}

Pomegranates were obtained from local supermarkets, and were of Spanish origin. $\mathrm{Zn}$ (II) as zinc sulphate heptahydrate $\left(\mathrm{ZnSO}_{4} \cdot 7 \mathrm{H}_{2} \mathrm{O}\right)$, potassium hydrogen phthalate HPLCgrade methanol and HPLC-grade water were purchased from ThermoFisher Scientific (Loughborough, UK). Punicalagin ( $\geq 98 \%)$, ascorbic acid and sodium carbonate $\left(\mathrm{Na}_{2} \mathrm{CO}_{3}\right)$ were purchased from Sigma-Aldrich (Gillingham, UK). Mueller-Hinton broth (MH broth) and Mueller-Hinton agar (MH agar) were both purchased from Oxoid (Basingstoke, UK).

\subsection{PRE Preparation and Evaluation}

PRE was produced by the hot aqueous extraction method, followed by freeze drying $[19,20]$. Pomegranates of Spanish origin were bought from a local supermarket and the rinds were excised then cut into strips prior to blending in deionized water $25 \% \mathrm{w} / \mathrm{v}$. This was boiled for $10 \mathrm{~min}$ before being centrifuged for 40 min on 6 occasions using Heraeus ${ }^{\mathrm{TM}}$ Multifuge $3 \mathrm{~S}-\mathrm{R}$ centrifuge at $5980 \times \mathrm{g}$. Next, the solution was vacuum filtered through $0.45 \mu \mathrm{m}$ nylon membrane membrane. The total volume of the filtrate was $289.5 \mathrm{~mL}$ which was then freeze-dried using a Scanivac ${ }^{\mathrm{TM}}$ freeze drier before being stored at $-20^{\circ} \mathrm{C}$. The freeze-dried PRE was reconstituted with phthalate buffer $(\mathrm{pH} 4.5)$ as required.

Quantitative analysis of PRE and punicalagin by reverse-phase HPLC using an Agilent

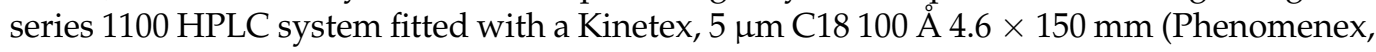
Macclesfield, UK) was used, along with a binary gradient elution programme involving A: methanol with $0.1 \%$ trifluoroacetic acid (TFA) and B: deionised water with $0.1 \%$ TFA (Table 1). Analyte detection was by UV at $258 \mathrm{~nm}$ and the analysis was performed at room temperature. Injection volume was $20 \mu \mathrm{L}$ and the flow rate was $1.5 \mathrm{~mL} / \mathrm{min}$; the total run time was $30 \mathrm{~min}$.

Table 1. Timetable for the HPLC elution of PRE, using a binary gradient elution program. A: methanol $+0.1 \%$ trifluoroacetic acid; B: water $+0.1 \%$ trifluoroacetic acid.

\begin{tabular}{ccc}
\hline Time $(\mathbf{m i n})$ & A. $\% \mathbf{M e O H}+\mathbf{0 . 1} \%$ TFA & B. $\% \mathbf{H}_{\mathbf{2}} \mathbf{O}+\mathbf{0 . 1} \% \mathbf{~ T F A ~}$ \\
\hline 0 & 5 & 95 \\
7 & 10 & 90 \\
15 & 20 & 80 \\
20 & 40 & 60 \\
25 & 60 & 40 \\
30 & 5 & 95 \\
\hline
\end{tabular}




\subsection{Microbiological Evaluations}

Four bacteria were used for investigation used in this study: MRSA (NCTC 12493), S. epidermidis ATCC 14990 (NCTC 11047), E. coli (NCTC 12923), and P. aeruginosa (NCTC 6750). Checkboard analysis was performed using standard techniques [20]. Time-kill assays were performed independently by two researchers on all four bacteria, using two different preparations of PRE.

Solutions of test substance in $990 \mu \mathrm{L}$ phthalate buffer (and negative control phthalate buffer only) were added to a $10 \mu \mathrm{L}$ aliquot of $0.5 \mathrm{McF}$ arland standard of tested bacteria in a $2 \mathrm{~mL}$ Eppendorf vial. After vortex mixing, incubation took place for different time points: 5, 10, and $20 \mathrm{~min}$; and additionally $60 \mathrm{~min}$ for MRSA and S. epidermidis. After each time-point, $100 \mu \mathrm{L}$ aliquots were transferred to $900 \mu \mathrm{L}$ of neutralizing agent (universal quenching agent, UQA) which was added to all experimental groups (including negative control) to halt the antimicrobial action of compounds and their combination and vortex mixed. After $60 \mathrm{~min}$, serial dilutions of the neutralized mixture were prepared out up to 8 times. Next, 3 drops in $10 \mu \mathrm{L}$ volume of each dilution were transferred to an MHA plate and incubated under aerobic conditions at $37^{\circ} \mathrm{C}$ for $24 \mathrm{~h}$. The number of surviving colonies were then enumerated [21]; and the following formula used to calculate the colony forming units per one millilitre $(\mathrm{CFU} / \mathrm{mL}$ ): (no of colonies $\times$ dilution factor)/volume of culture media

The $\log _{10}$ reduction in CFU $/ 1 \mathrm{~mL}$ was determined versus control (phthalate buffer, $\mathrm{pH}$ 4.5) using the following Equation (1), where $\mathrm{A}$ is $\mathrm{CFU} / \mathrm{mL}$ of the control (phthalate buffer) and $\mathrm{B}$ is $\mathrm{CFU} / \mathrm{mL}$ of test sample.

$$
\llbracket \log \rrbracket \_10(\mathrm{~A})-\llbracket \log \rrbracket \_10(\mathrm{~B})
$$

\subsection{Statistical Analysis}

Experiments were performed by two researchers using two different PRE preparations, and each researcher repeated experiment nine times for each microbe. Then results from each researcher were collected and data presented as mean \pm standard deviation (SD). GraphPad Prism 8.0 software (GraphPad Software, San Diego, CA, USA) was used for statistical analysis: one-way ANOVA was performed with post-test Tukey correction: $p<0.05$ was considered statistically significant and in the graphical plots denoted as ${ }^{*} p \leq 0.05,{ }^{* *} p \leq 0.01,{ }^{* * *} p \leq 0.001$, and ${ }^{* * * *} p \leq 0.0001$.

\subsection{Computational Surface Charge Analysis}

All molecular modelling experiments were performed on Asus WS X299 PRO Intel ${ }^{\circledR}$ i9-10980XE CPU @ $3.00 \mathrm{GHz} \times 36$ running Ubuntu 18.04. The molecular structures were prepared by MOE QuikPrep tool generating possible ionization states at $\mathrm{pH} 7.4$ and $\mathrm{pH}$ 4.5. Quantitative calculation of formal charge and relative negative charges were calculated at both $\mathrm{pH}$ values (Molecular operating environment (MOE), Montreal, QC, Canada).

\section{Results}

\subsection{PRE Evaluation}

The chromatogram shown in Figure 1 highlights the multiple tannin compounds present in PRE and-in particular-punicalagin, which exists as two anomers in a characteristic 1:2 ratio: $\alpha$-punicalagin with retention time of $7.38 \mathrm{~min}$ and anomer $\beta$-punicalagin with retention time $11.74 \mathrm{~min}$. The identity of punicalagin was also confirmed of punicalagin by comparison with purified punicalagin. Spiking with standard punicalagin gave rise to both $\alpha$ and $\beta$ punicalagin peaks, and this was also used to prepare a standard calibration curve from which it was determined that the mean punicalagin level in the 2 extracts was PRE was $21.7 \%$ (1 mg in each $4.61 \mathrm{mg}$ of PRE). The level of punicalagin, which is known to be the main active component of PRE, is provided to allow a comparison with other papers. 


\subsection{MRSA}

Checkerboard analysis indicated synergistic activity between PRE and Zn (II) against MRSA, with a FIC index of $<0.5$ (data not shown). Figure 2A shows the log reduction of MRSA CFUs with PRE $(1 \mathrm{mg} / \mathrm{mL})$ and $\mathrm{Zn}$ (II) $(0.5 \mathrm{M})$ alone and in combination, up to $1 \mathrm{~h}$ incubation time. Despite negligible changes in MRSA CFUs were identified with PRE alone over the $1 \mathrm{~h}$ time course $(p>0.05)$, the combination of PRE with $\mathrm{Zn}$ (II) induced significant log reductions in CFUs compared to PRE alone, at all time-points analyzed $(p<0.0001-0.001)$. Although $\mathrm{Zn}$ (II) alone also induced significant $\log$ reductions in CFUs compared to PRE alone at $20 \mathrm{~min}(p<0.0001)$, these responses were to a lower magnitude than with PRE and Zn (II) combined, given the significantly greater log reductions induced by the combinational treatment $(p<0.0001)$. No discernible differences between $0.5 \mathrm{M} \mathrm{Zn}$ (II) and the combined treatment were evident at $1 \mathrm{~h}(p>0.05)$, as the maximum kill had likely occurred.

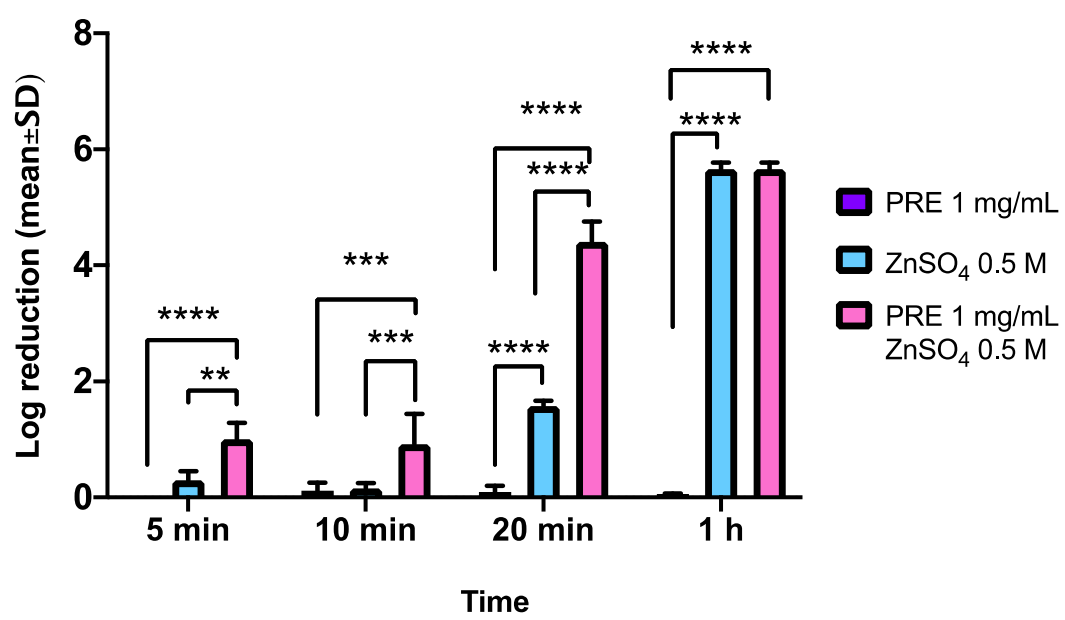

(A)

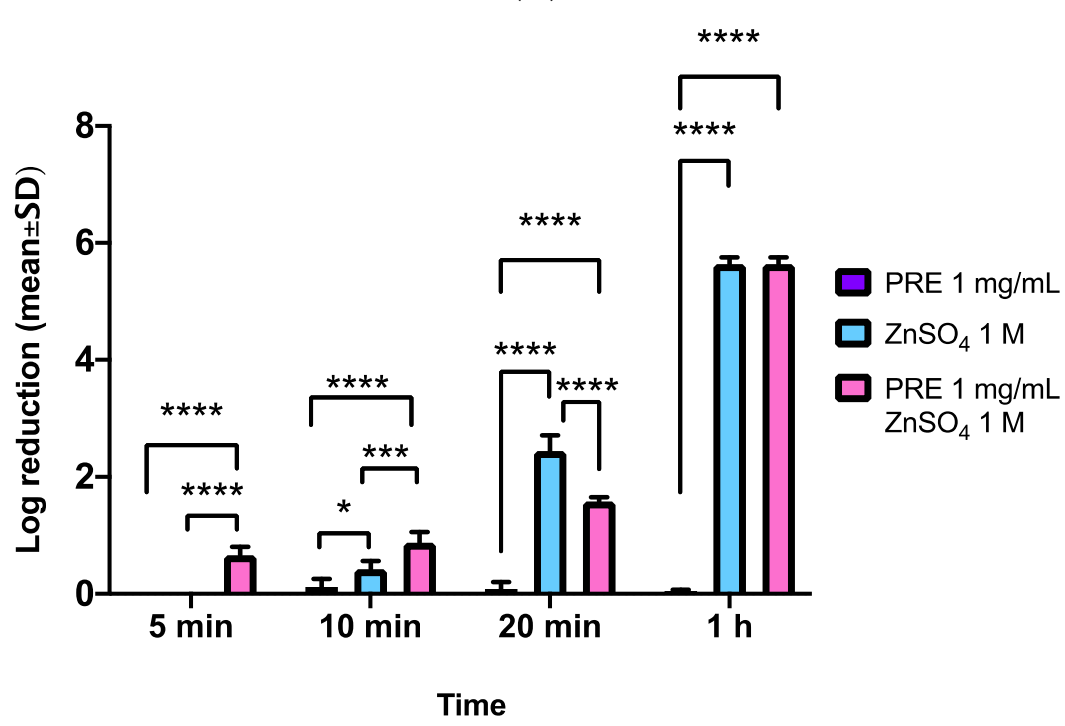

(B)

Figure 2. $\log _{10}$ reductions in MRSA CFUs at incubation times of $5 \mathrm{~min}, 10 \mathrm{~min}, 20 \mathrm{~min}$, and $1 \mathrm{~h}$ $(n=9 \pm \mathrm{SD})$. (A) PRE (1 mg/mL), and Zn (II) (0.5 M) alone and in combination. (B) PRE (1 mg/mL), Zn (II) (1 M) alone, and in combination. Tukey's multiple comparison post-hoc test show statistically significant differences $\left({ }^{*} p \leq 0.05,{ }^{* *} p \leq 0.01,{ }^{* * *} p \leq 0.001,{ }^{* * * *} p \leq 0.0001\right)$. 
Further analyses involving PRE $(1 \mathrm{mg} / \mathrm{mL})$ with higher concentrations of $\mathrm{Zn}$ (II) $(1 \mathrm{M})$ alone and in combination, up to $1 \mathrm{~h}$ incubation time, are shown in Figure 2B. PRE alone again evoked negligible changes in MRSA CFUs over the $1 \mathrm{~h}$ time course $(p>0.05)$. The combination of PRE with Zn (II) induced further significant log reductions in CFUs compared to PRE alone, at all time-points analysed (all $p<0.0001$ ). Zn (II) alone again induced significant log reductions in CFUs compared to PRE alone, although given the higher concentrations used (1 M), significant Zn (II) effects were shown at $10 \mathrm{~min}, 20 \mathrm{~min}$, and $1 \mathrm{~h}$, compared to PRE alone $(p<0.0001-0.05)$. However, these responses were more equivalent to those with PRE and Zn (II) combined, as although significantly greater log reductions induced by the combinational treatment at $10 \mathrm{~min}(p<0.001)$, Zn (II) alone promoted significantly larger reductions in MRSA CFUs at $20 \mathrm{~min}(p<0.0001)$. Again, no obvious differences between $1 \mathrm{M} \mathrm{Zn}$ (II) and the combined treatment were evident at $1 \mathrm{~h}$ ( $p>0.05)$, as the maximum kill had likely occurred. Thus, although such findings implied that PRE and Zn (II) exerted a synergistic effect on MRSA CFUs, this was more apparent at $\mathrm{Zn}$ (II) concentrations of $0.5 \mathrm{M}$, as the synergy became less evident as $\mathrm{Zn}$ (II) concentrations increased $(1 \mathrm{M})$.

\subsection{S. epidermidis}

Checkerboard analysis revealed synergistic activity between PRE and Zn (II) against S. epidermidis, with a FIC index of $<0.5$ (data not shown). For S. epidermidis the concentration range of $\mathrm{Zn}$ (II) was expanded to include the lower concentrations of $0.25 \mathrm{M}$ and $0.125 \mathrm{M}$ over maximum incubation time of $1 \mathrm{~h}$. As above, negligible changes in S. epidermidis CFUs were identified with PRE alone over the $1 \mathrm{~h}$ time course $(p>0.05)$. However, the combination of PRE with $\mathrm{Zn}$ (II) resulted in significant log reductions in CFUs compared to PRE alone at $1 \mathrm{~h}$ with $0.125 \mathrm{M} \mathrm{Zn} \mathrm{(II)} \mathrm{(} p<0.0001$, Figure $3 \mathrm{~A}), 20 \mathrm{~min}$ and $1 \mathrm{~h}$ with $0.25 \mathrm{M} \mathrm{Zn}$ (II) (both $p<0.0001$, Figure 3B), $10 \mathrm{~min}, 20 \mathrm{~min}$, and $1 \mathrm{~h}$ with $0.5 \mathrm{M} \mathrm{Zn}$ (II) $(p<0.0001-0.001$, Figure 3C); and all time-points with $1 \mathrm{M}$ Zn (II) ( $p<0.0001-0.05$, Figure 3D). Zn (II) alone again induced significant log reductions in CFUs compared to PRE alone at $1 \mathrm{~h}$ with $0.125 \mathrm{M}, 0.25 \mathrm{M}$ and 0.5 M Zn (II) (all $p<0.0001$, Figure 3A-C), although higher Zn (II) concentrations $(1 \mathrm{M})$ promoted significant log reductions in CFUs compared to PRE alone over wider timeframes of $10 \mathrm{~min}, 20 \mathrm{~min}$, and $1 \mathrm{~h}(p<0.0001-0.05$, Figure 3D). However, these responses were at a lower extent than those induced with PRE and Zn (II) combined, given the significantly greater log reductions induced by the combinational treatment overall, at $1 \mathrm{~h}$ with $0.125 \mathrm{M} \mathrm{Zn}$ (II) ( $p<0.0001$, Figure $3 \mathrm{~A}$ ), $20 \mathrm{~min}$ and $1 \mathrm{~h}$ with $0.25 \mathrm{M} \mathrm{Zn}$ (II) ( $p<0.0001-0.01$, Figure 3B), $10 \mathrm{~min}, 20 \mathrm{~min}$, and $1 \mathrm{~h}$ with $0.5 \mathrm{M} \mathrm{Zn} \mathrm{(II)}(p<0.0001-0.01$, Figure 3C); and at $10 \mathrm{~min}$ and $20 \mathrm{~min}$ with $1 \mathrm{M} \mathrm{Zn}$ (II) (both $p<0.0001$, Figure 3D). No apparent differences between the $0.5 \mathrm{M}$ and $1 \mathrm{M}$ Zn (II) concentrations alone with the combined treatment were evident at $1 \mathrm{~h}(p>0.05)$, as the maximum kill had likely occurred. 

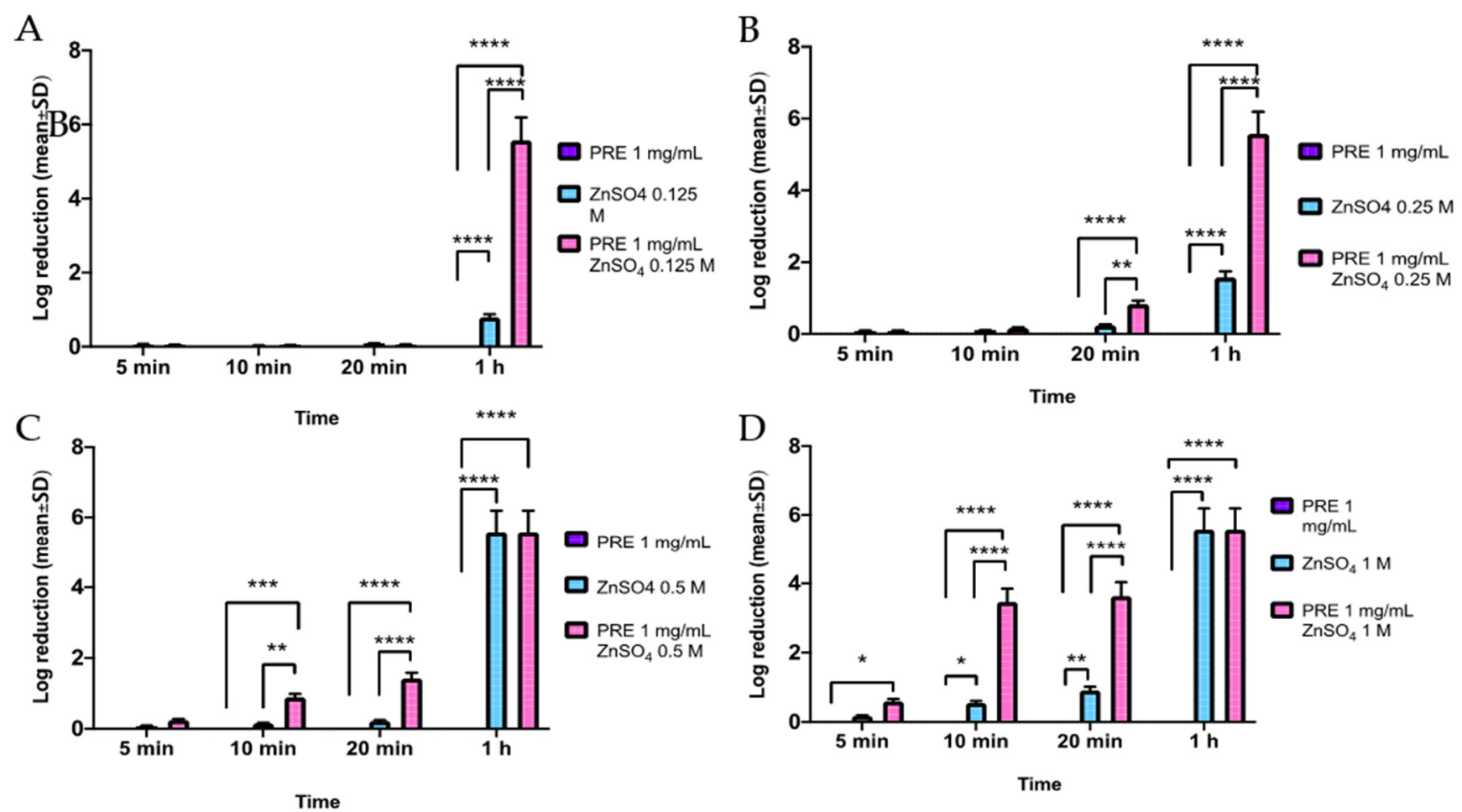

Figure 3. $\log _{10}$ reduction of $S$. epidermidis CFUs at incubation times of $5 \mathrm{~min}, 10 \mathrm{~min}, 20 \mathrm{~min}$ and $1 \mathrm{~h}(n=9 \pm \mathrm{SD})$. (A) PRE ( $1 \mathrm{mg} / \mathrm{mL})$ and $\mathrm{Zn}$ (II) $(0.125 \mathrm{M})$, alone and in combination. (B) PRE (1 mg/mL) and Zn (II) (0.25 M) alone and in combination. (C) PRE ( $1 \mathrm{mg} / \mathrm{mL})$ and $\mathrm{Zn}$ (II) $(0.5 \mathrm{M})$ alone and in combination. (D) PRE (1 mg/mL) and Zn (II) (1 M) alone and in combination. Tukey's multiple comparison post-hoc test show statistically significant differences $\left({ }^{*} p \leq 0.05\right.$, $\left.{ }^{* *} p \leq 0.01,{ }^{* * *} p \leq 0.001,{ }^{* * * *} p \leq 0.0001\right)$.

\subsection{E. coli}

Checkerboard analysis of PRE and Zn (II) effects on E. coli demonstrated that the log reductions in CFUs achieved with PRE $(1 \mathrm{mg} / \mathrm{mL})$ alone were more apparent over the 20-min time course, than evident with MRSA and S. epidermidis (Figure 4A,B). However, the combination of PRE with $0.5 \mathrm{M} Z \mathrm{Zn}$ (II) induced significantly greater log reductions in CFUs compared to PRE alone, at all time-points analysed (all $p<0.0001$, Figure 4A). Similarly, despite Zn (II) alone also inducing significant log reductions in CFUs compared to PRE alone at $5 \mathrm{~min}$ and $20 \mathrm{~min}$ (both $p<0.0001$ ), the magnitude of the log reductions in CFUs were again to a lesser extent than with the combinational treatment (all $p<0.0001)$.

Increasing the $\mathrm{Zn}$ (II) concentration to $1 \mathrm{M}$ (with PRE $1 \mathrm{mg} / \mathrm{mL}$ ), produced further, more rapid log reductions in CFUs, than PRE and 0.5 M Zn (II) (Figure 4B). PRE alone induced comparable log reduction to Figure $4 \mathrm{~A}(p>0.05)$, although the combination of PRE with $1 \mathrm{M}$ Zn (II) induced further significant log reductions in CFUs compared to PRE alone, at all time-points analysed (all $p<0.0001$ ). Zn (II) alone also induced significant log reductions in CFUs compared to PRE alone at all time-points (all $p<0.0001)$. However, the combinational treatment was shown to promote much greater log reductions in CFUs, compared to Zn (II) alone, at all time-points (all $p<0.0001$ ). 


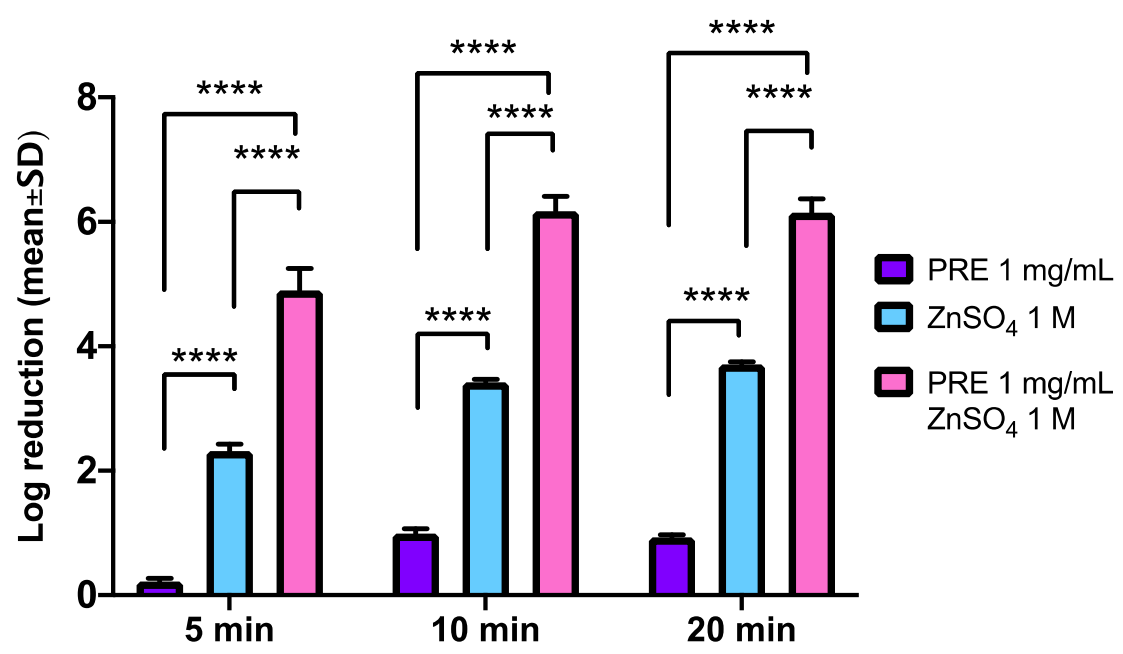

Time

(A)

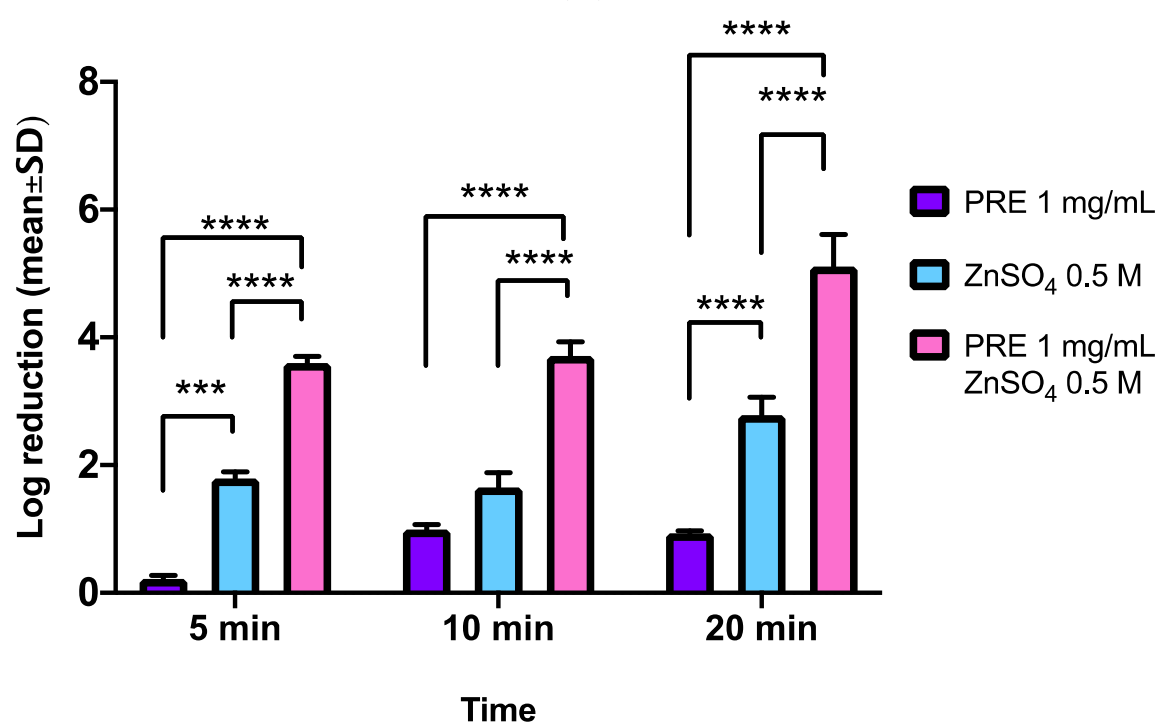

(B)

Figure 4. $\log _{10}$ reduction of $E$. coli CFUs at incubation times of $5 \mathrm{~min}, 10 \mathrm{~min}$ and $20 \mathrm{~min}(n=9 \pm \mathrm{SD})$. (A) PRE (1 mg/mL) and Zn (II) (0.5 M) alone and in combination. (B) PRE (1 mg/mL) and Zn (II) (1 M) alone and in combination. Tukey's multiple comparison post-hoc test show statistically significant differences $\left(* * * \leq 0.001,{ }^{* * * *} p \leq 0.0001\right)$.

\subsection{P. aeruginosa}

Checkerboard analysis of PRE and Zn (II) effects on P. aeruginosa revealed that minor $\log$ reduction changes in CFUs were achieved with PRE $(1 \mathrm{mg} / \mathrm{mL})$ alone over the $20 \mathrm{~min}$ time course (Figure 5A,B). However, the combination of PRE with $0.5 \mathrm{M} Z \mathrm{Zn}$ (II) produced significantly greater log reductions in CFUs compared to PRE alone, at all time-points analysed (all $p<0.0001$, Figure 5A). Although Zn (II) alone also induced significant log reductions in CFUs compared to PRE alone at $5 \mathrm{~min}$ and $10 \mathrm{~min}$ (both $p<0.0001$ ), the log reductions in CFUs were again much larger with the combinational treatment (all $p<0.0001)$. 


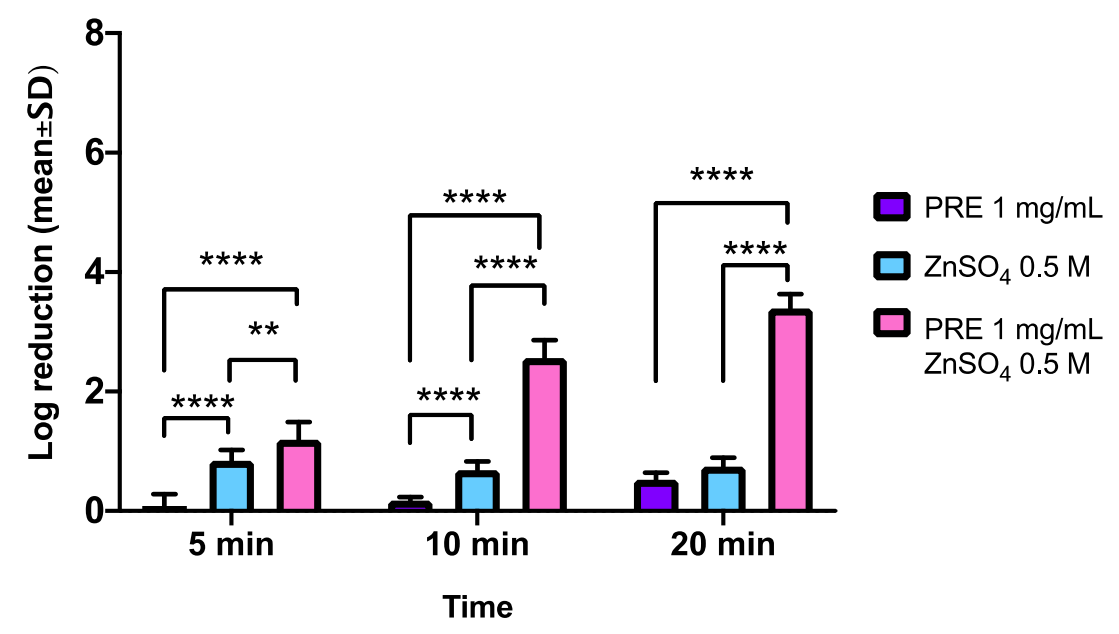

(A)

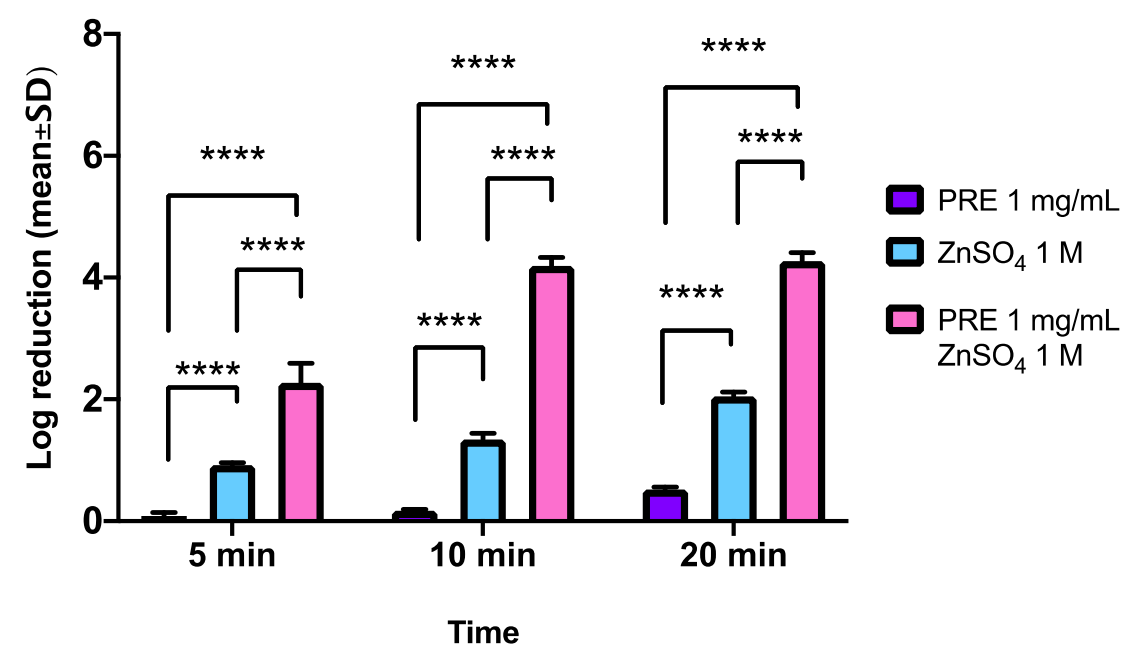

(B)

Figure 5. $\log _{10}$ reduction of $P$. aeruginosa CFUs at incubation times of $5 \mathrm{~min}, 10 \mathrm{~min}$, and $20 \mathrm{~min}$ $(n=9 \pm \mathrm{SD})$. (A) PRE (1 mg/mL) and Zn (II) (0.5 M) alone and in combination. (B) PRE ( $1 \mathrm{mg} / \mathrm{mL})$ and $\mathrm{Zn}$ (II) (1 M) alone and in combination. Tukey's multiple comparison post-hoc test show statistically significant differences $\left.{ }^{* *} p \leq 0.01,{ }^{* * * *} p \leq 0.0001\right)$.

Additional increases in $\mathrm{Zn}$ (II) concentration (1 M) with PRE (1 mg/mL) also promoted more rapid log reductions in CFUs, than PRE and 0.5 M Zn (II) (Figure 5B). PRE alone induced similar log reduction to Figure $5 \mathrm{~A}(p>0.05)$, although the combination of PRE with $1 \mathrm{M}$ Zn (II) induced further significant log reductions in CFUs compared to PRE alone at all time-points analysed (all $p<0.0001$ ). Zn (II) alone also induced significant log reductions in CFUs compared to PRE alone at all time-points (all $p<0.0001)$. However, the combinational treatment was shown to promote much greater log reductions in CFUs, compared to Zn (II) alone, at all time-points (all $p<0.0001$ ).

\section{Discussion}

The main scope of this paper was to use PRE/Zn (II) combinations that had shown synergy against another microbes and establish whether it also has efficacy against the four bacteria in the panel — the aim being to work towards the development of a single product that is broad spectrum in its activity. Combination therapy-i.e., the enhancement of the potency of an antimicrobial agent by the simultaneous administration of a second agent-is of increasing interest in the hunt for new approaches to combat anti-microbial resistance. Here, we use the term 'synergy', rather than 'potentiation', to describe such modulation, 
as both PRE and-in particular-Zn (II) show levels of activity against microbes when supplemented individually. However, the data suggests that PRE may in fact potentiate the anti-microbial activity of $\mathrm{Zn}$ (II) overall.

PRE is known to possess antimicrobial properties and its combination with metal ions has been shown to result in the significant enhancement of such activities. Potentiation of PRE with Fe (II) was found to produce an 11-log reduction in bacteriophage [17], although the enhancement was temporary and its cessation coincided with solution blackening, as Fe (III) was oxidised to Fe (III). The copper (II) ions have also been proposed as a potentiating agent and have shown to be more effective in combination with PRE than Fe (II) and also $\mathrm{Zn}$ (II) [18], although the incubation times were shorter than those reported herein. However, $\mathrm{Cu}$ (II) is associated with high toxicity when administered to human cells, which means it use as a novel anti-microbial drug would be limited [22,23]. The Zn (II) ion, which has the benefit of lower toxicity, led to significant synergistic microbicidal activity when combined with PRE against HSV, which was not time-limited [24]. These findings were more recently reflected in the developed synergistic anti-microbial activity of PRE and Zn (II) against the bacterium, M. luteus [20].

In order to work towards a single broad-spectrum microbicidal product, higher concentrations were used in order to generally align with the viricidal data reported previously against HSV-1 and HSV-2 (Houston et al., 2017) [19]. Here, we sought to examine such effects against a wide panel of bacteria of a more pathogenic nature: MRSA [25], S. epidermidis [26], E. coli [27], and P. aeruginosa [28]. These species are responsible for a diversity of serious topical and GI infections, as described in the citations. To establish reproducibility, two sets of data were obtained independently by two researchers, using PRE prepared on two separate occasions with different PRE extracts. Results, presented as global means based upon two data sets, show statistically significant synergistic bactericidal activity for all four bacterial species investigated, although different levels of sensitivity to the PRE and Zn (II) combination were apparent. Additionally, higher levels of Zn (II) generally led to synergism occurring more rapidly, possibly as a consequence of the increased thermodynamic activity of the compounds in solution. An earlier paper reported significant log reductions in S. aureus, E. coli, and P. aeruginosa were found when PRE was applied with $\mathrm{Cu}$ (II), but not when Zn (II) was used [29].

Mechanistically, the synergistic antimicrobial activity observed between PRE and Zn (II) has yet to be fully elucidated, it is likely that Zn (II) and components of PRE such as punicalagin, are acting independently, albeit cooperatively. It has previously been suggested that PRE may show enhanced activity due to redox cycling of co-administered metal ions, such as Fe (II) and $\mathrm{Cu}$ (II), which would increase local levels of reactive oxygen species (ROS) [18]. However, $\mathrm{Zn}$ (II) is a stable ion that is not known to undergo such redox processes. Mechanistically, punicalagin has been reported to damage the integrity of bacterial membranes. Electron microscopy observations showed that the cell membrane structures of Salmonella typhimurium were damaged after treatment with punicalagin, inducing an increase in the extracellular concentrations of potassium and a release of cell constituents [9]. Although it is likely that, as the predominant tannin, punicalagin would exert a major membrane destabilising effect, other tannins and constituents present in lower proportions may further anti-microbial exert effects. As $\mathrm{Zn}$ (II) is also known to be toxic to bacteria at certain concentrations possibly due to blocking Mn (II) uptake [30], it can also be postulated that membrane damage caused by punicalagin would allow greater influx of $\mathrm{Zn}$ (II) to toxic levels, due to compromised efflux pumping.

Here we also observed that Gram-negative E. coli and P. aeruginosa were notably less sensitive than the Gram-positive MRSA and S. epidermidis. The cell walls of Gram-positive bacteria are predominantly composed of peptidoglycan, whereas the cell walls of Gramnegative bacteria are more complex, with the presence of a plasma outer membrane located outside of the peptidoglycan layers [31]. This layer presents an additional diffusional barrier for the penetration of polar compounds, such as large hydrolysable tannins in PRE, before the outer membrane bilayer core can be reached [32,33]. Additionally, bac- 
terial cell walls generally possess a negative charge at physiological $\mathrm{pH}$ [34], although there is variation between species. In Gram-positive bacteria surface charge is due to the presence of teichoic acids linked to either the peptidoglycan or to the underlying plasma membrane- these teichoic acids are negatively charged because of presence of phosphate in their structure. Gram-negative bacteria have an outer covering of phospholipids and lipopolysaccharides, which impart a strongly negative charge to surface of Gram-negative bacterial cells. However, under more acidic conditions-such as $\mathrm{pH} 4.5$, as used in this work-such charges would be suppressed leading to surface charge neutrality. Similarly, the charge of tannin components of PRE is also suppressed at $\mathrm{pH} 4.5$ relative to $\mathrm{pH} 7.4$ (Figure 6). One immediately apparent effect of such neutralisation is the loss of electrostatic repulsion between the bacterial surface charges and tannins would make interaction between them more favourable, potentially leading to the damage as reported previously [9]. In the absence of functioning efflux pumps the very small cationic $\mathrm{Zn}$ (II) would be able to penetrate the bacteria to toxic levels and accelerate cell death.

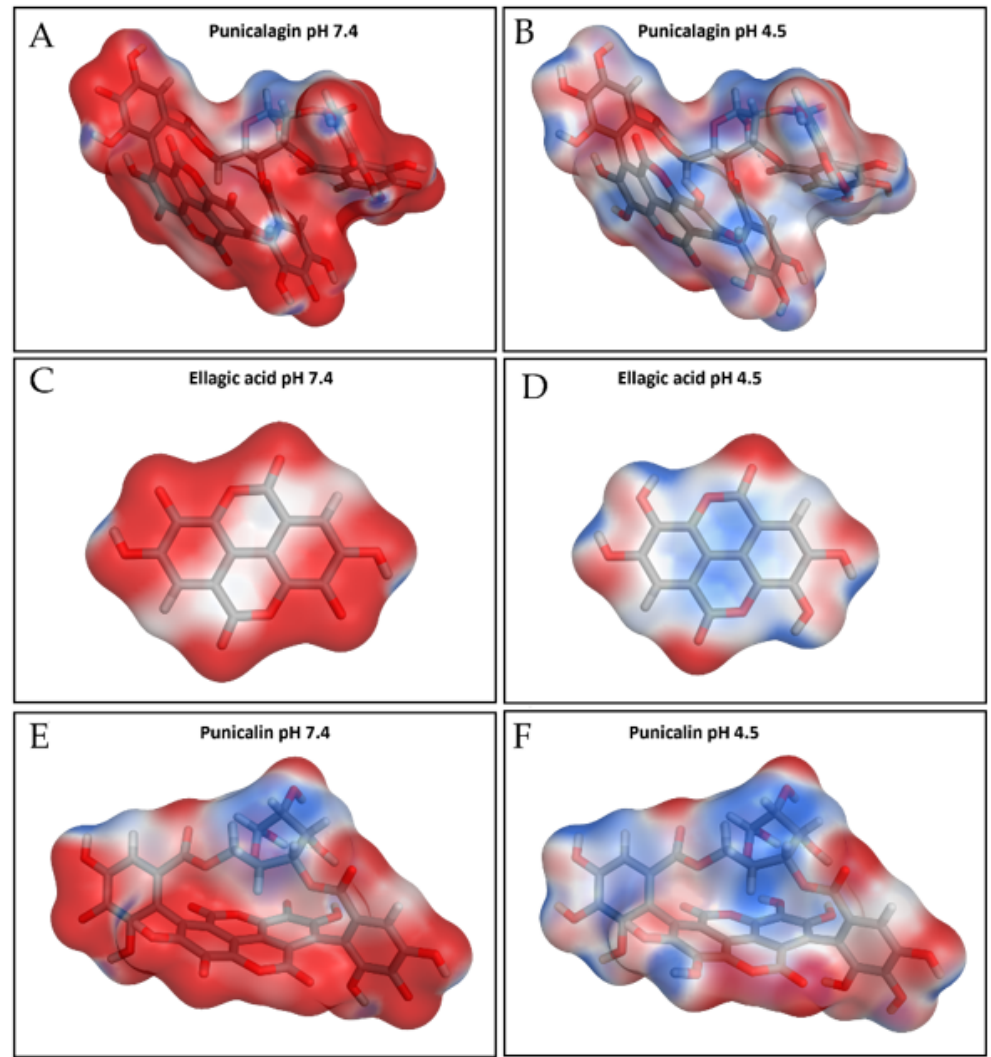

\begin{tabular}{|l|c|c|c|c|}
\hline Tannin & $\begin{array}{c}\text { Formal charge } \\
\mathrm{pH} 7.4\end{array}$ & $\begin{array}{c}\text { Relative negative charge } \\
\mathrm{pH} 7.4\end{array}$ & $\begin{array}{c}\text { Formal charge } \\
\mathrm{pH} 4.5\end{array}$ & $\begin{array}{c}\text { Relative negative charge } \\
\mathrm{pH} 4.5\end{array}$ \\
\hline Punicalagin & -5 & 0.0657 & 0 & 0.0373 \\
\hline Ellagic acid & -2 & 0.2227 & 0 & 0.1420 \\
\hline Punicalin & -4 & 0.0870 & 0 & 0.0532 \\
\hline
\end{tabular}

Figure 6. Electrostatic surface maps of punicalagin, ellagic acid, and punicalin at $\mathrm{pH} 7.4$ and $\mathrm{pH}$ 4.5. Punicalagin, like other tannins, possesses a negative charge at $\mathrm{pH} 7.4$; however, they are all neutral with almost equal distribution of electrostatic charge positive and negative at $\mathrm{pH}$ 4.5. The areas colored in red and blue represent respectively negative and positive regions of the electrostatic potential. Table contains computed charges for punicalagin, ellagic acid, and punicalin, confirming absence of charge at $\mathrm{pH} 4.5$. 
Taken alongside the data obtained previously for HSV and M. luteus, based on these collective findings, there is justification for the development of PRE/Zn (II) as a wide spectrum anti-microbial therapeutic system. This is further supported by the anti-inflammatory properties of PRE/Zn (II) against the local arachidonic acid inflammation pathway [35], antioxidant capacities, and wound healing potential [36].

Topical drug delivery generally involves administering lower doses as they are applied locally to the required site, rather than systemically where effects such as a large volume of distribution must be accounted for. The topical route of administration, such as via a gel or spray, is most appropriate for this system because unlike cases such as co-amoxiclav where the two drugs act independently, the synergistic activity observed with PRE and Zn (II) arises through concentration-dependent co-operative interplay that is unlikely to occur following a systemically administered dose. Furthermore, the levels of $\mathrm{Zn}$ (II) used here are high, which precludes them from systemic administration. However, topical cream products containing zinc sulfate are available. Sharquie et al. (2008) used $10 \%(0.35 \mathrm{M})$ solutions to examine the effects of zinc sulfate on melasma in a clinical trial—in addition to good clinical responses, no side effects were reported apart from a mild stinging sensation reported in a few patients [37]. Furthermore, the same group later used a 25\% $(0.87 \mathrm{M})$ zinc sulphate solution to determine effects on actinic keratosis in 100 patients-again, good clinical responses were observed and the treatment was not associated with side effects apart from mild and transient burning sensation which was encountered in the open lesions of one-third of the patient cohort [38]. The levels of zinc sulfate used in our formulations are similar to these two clinical studies. Moreover, Sharquie et al. (2012) proposed using even higher concentrations of zinc sulfate for future studies [38]. Topical application of zinc in high levels also has a history of use in treating eczemas including contact dermatitis [39].

\section{Conclusions}

The findings in this paper provide further evidence to support the development of a novel, broad-spectrum, anti-microbial product based upon the synergistic microbicidal activity PRE and Zn (II). Such a product would be of value in combating multi-drug, anti-microbial resistance worldwide.

Author Contributions: Conceptualization, C.M.H. and D.M.J.H.; Formal analysis, D.M.J.H., V.C., N.H., C.M., A.A., M.J., C.M.H. and C.V.; Funding acquisition, C.M.H.; Investigation, D.M.J.H., V.C., N.H., C.M., A.A., M.J., C.M.H. and C.V.; Methodology, D.M.J.H., V.C., C.M.H., R.M. and C.V.; Project administration, C.M.H.; Supervision, D.M.J.H., C.M.H., V.C. and R.M.; Writing-original draft, D.M.J.H., N.H., C.M., A.A., M.J. and C.M.H.; Writing-review and editing, C.M.H., V.C., R.M. and C.V. All authors have read and agreed to the published version of the manuscript.

Funding: This research received no external funding.

Institutional Review Board Statement: Not applicable.

Informed Consent Statement: Not applicable.

Data Availability Statement: Not applicable.

Conflicts of Interest: The authors declare no conflict of interest.

\section{References}

1. Ventola, C.L. The antibiotic resistance crisis: Part 1: Causes and threats. Pharm. Ther. 2015, 40, $277-283$.

2. WHO. Antimicrobial Resistance. 2020. Available online: https://www.who.int/news-room/fact-sheets/detail/antimicrobialresistance (accessed on 12 December 2021).

3. Vilar-Compte, D.; Camacho-Ortiz, A.; Ponce-de-León, S. Infection control in limited resources countries: Challenges and priorities. Curr. Infect. Dis. Rep. 2017, 19, 20. [CrossRef]

4. Celiksoy, V.; Heard, C.M. The antimicrobial potential of pomegranate. In Pomegranate; InfoTechOpen: London, UK, 2021; Available online: https:/ / cdn.intechopen.com/pdfs/75059.pdf (accessed on 13 December 2021).

5. Singh, B.; Singh, J.P.; Kaur, A.; Singh, N. Phenolic compounds as beneficial phytochemicals in pomegranate (Punica granatum L.) peel: A review. Food Chem. 2018, 261, 75-86. [CrossRef] [PubMed] 
6. Duman, A.D.; Ozgen, M.; Dayisoylu, K.S.; Erbil, N.; Durgac, C. Antimicrobial activity of six pomegranate (Punica granatum L.) varieties and their relation to some of their pomological and phytonutrient characteristics. Molecules 2009, 14, $1808-1817$. [CrossRef] [PubMed]

7. Al-Zoreky, N.S. Antimicrobial activity of pomegranate (Punica granatum L.) fruit peels. Int. J. Food Microbiol. 2002, 134, 244-248. [CrossRef]

8. Nozohour, Y.; Golmohammadi, R.; Mirnejad, R.; Fartashvand, M. Antibacterial activity of pomegranate (Punica granatum 1.) seed and peel alcoholic extracts on Staphylococcus aureus and Pseudomonas aeruginosa isolated from health centers. J. Appl. Biotechnol. Rep. 2018, 5, 32-36. [CrossRef]

9. Li, G.; Xu, Y.; Pan, L.; Xia, X. Punicalagin damages the membrane of Salmonella typhimurium. J. Food Prot. 2020, 83, 2102-2106. [CrossRef]

10. Mousavi, S.; Weschka, D.; Bereswill, S.; Heimesaat, M.M. Preclinical evaluation of oral urolithin-a for the treatment of acute campylobacteriosis in campylobacter jejuni infected microbiota-depleted IL-10-/- mice. Pathogens 2020, 10, 7. [CrossRef]

11. Fischbach, M.A. Combination therapies for combating antimicrobial resistance. Curr. Opin. Microbiol. 2011, 14, 519-523. [CrossRef]

12. Schmid, A.; Wolfensberger, A.; Nemeth, J.; Schreiber, P.W.; Sax, H.; Kuster, S.P. Monotherapy versus combination therapy for multidrug-resistant Gram-negative infections: Systematic review and meta-analysis. Sci. Rep. 2019, 9, 15290. [CrossRef]

13. Lee, Y.; Puumala, E.; Robbins, N.; Cowen, L.E. Antifungal drug resistance: Molecular mechanisms in Candida albicans and beyond. Chem. Rev. 2021, 121, 3390-3411. [CrossRef]

14. Khameneh, B.; Diab, R.; Ghazvini, K.; Bazzaz, B.S.F. Breakthroughs in bacterial resistance mechanisms and the potential ways to combat them. Microb. Pathog. 2016, 95, 32-42. [CrossRef]

15. Woerther, P.-L.; Burdet, C.; Chachaty, E.; Andremont, A. Trends in human fecal carriage of extended-spectrum $\beta$-lactamases in the community: Toward the globalization of CTX-M. Clin. Microbiol. Rev. 2013, 26, 744-758. [CrossRef]

16. Khameneh, B.; Iranshahy, M.; Soheili, V.; Bazzaz, B.S.F. Review on plant antimicrobials: A mechanistic viewpoint. Antimicrob. Resist. Infect. Control 2019, 8, 118. [CrossRef]

17. Stewart, G.S.; Jassim, S.A.; Denyer, S.P.; Newby, P.; Linley, K.; Dhir, V.K. The specific and sensitive detection of bacterial pathogens within 4 h using bacteriophage amplification. J. Appl. Microbiol. 1998, 84, 777-783. [CrossRef] [PubMed]

18. Gould, S.W.J.; Fielder, M.D.; Kelly, A.F.; Naughton, D.P. Anti-microbial activities of pomegranate rind extracts: Enhancement by cupric sulphate against clinical isolates of S. aureus, MRSA and PVL positive CA-MSSA. BMC Complement. Altern. Med. 2009, 9, 23. [CrossRef] [PubMed]

19. Houston, D.M.; Bugert, J.J.; Denyer, S.P.; Heard, C.M. Potentiated virucidal activity of pomegranate rind extract (PRE) and punicalagin against Herpes simplex virus (HSV) when co-administered with zinc (II) ions, and antiviral activity of PRE against HSV and aciclovir-resistant HSV. PLoS ONE 2017, 12, e0188609.

20. Celiksoy, V.; Moses, R.L.; Sloan, A.J.; Moseley, R.; Heard, C.M. Synergistic in vitro antibacterial activity of pomegranate rind extract and zinc (II) against Micrococcus luteus under planktonic and biofilm conditions. Pharmaceutics 2021, 13, 851. [CrossRef]

21. Miles, A.A.; Misra, S.S. The estimation of the bactericidal power of the blood. J. Hyg. 1938, 38, 732-749. [CrossRef] [PubMed]

22. Hostynek, J.J.; Maibach, H.I. Skin irritation potential of copper compounds. Toxicol. Mech. Methods 2004, 14, 205-213. [CrossRef]

23. Li, H. Selected biomarkers revealed potential skin toxicity caused by certain copper compounds. Sci. Rep. 2016, 6, 37664. [CrossRef] [PubMed]

24. Houston, D.M.J. Towards a Nanomedicine-Based Virucidal System. Ph.D. Thesis, Cardiff University, Cardiff, UK, 2011.

25. Gordon, R.J.; Lowy, F.D. Pathogenesis of Methicillin-Resistant Staphylococcus aureus Infection. Clin. Infect. Dis. 2008, 46, S350-S359. [CrossRef]

26. Otto, M. Staphylococcus epidermidis-The 'accidental' pathogen. Nat. Rev. Microbiol. 2009, 7, 555-567. [CrossRef]

27. Smith, L.; Fratamico, P.M. Escherichia coli as a Pathogen. In Foodborne Diseases, 3rd ed.; Dodd, C.E.R., Aldsworth, T., Stein, R.A., Cliver, D.O., Riemann, H.P., Eds.; Academic Press: Cambridge, MA, USA, 2017; Chapter 7; pp. 189-208. ISBN 9780123850072.

28. Azam, M.W.; Khan, A.U. Updates on the pathogenicity status of Pseudomonas aeruginosa. Drug Discov. Today 2019, 24, 350-359. [CrossRef]

29. McCarrell, E.M.; Gould, S.W.J.; Fielder, M.D.; Kelly, A.F.; El Sankary, W.; Naughton, D.P. Antimicrobial activities of pomegranate rind extracts: Enhancement by addition of metal salts and vitamin C. BMC Complement. Altern. Med. 2008, 8, 64. [CrossRef]

30. David, R. Why zinc is bad for bacteria. Nat. Rev. Genet. 2011, 10, 4. [CrossRef]

31. Dörr, T.; Moynihan, P.J.; Mayer, C. Editorial: Bacterial Cell Wall Structure and Dynamics. Front. Microbiol. 2019, 10, 2051. [CrossRef]

32. Brown, D.G.; May-Dracka, T.L.; Gagnon, M.M.; Tommasi, R. Trends and exceptions of physical properties on antibacterial activity for Gram-positive and Gram-negative pathogens. J. Med. Chem. 2014, 57, 10144-10161. [CrossRef] [PubMed]

33. Wafa, B.A.; Makni, M.; Ammar, S.; Khannous, L.; Hassana, A.B.; Bouaziz, M.; Gdoura, R. Antimicrobial effect of the Tunisian Nana variety Punica granatum L. extracts against Salmonella enterica (serovars Kentucky and Enteritidis) isolated from chicken meat and phenolic composition of its peel extract. Int. J. Food Microbiol. 2017, 241, 123-131. [CrossRef] [PubMed]

34. Wilson, W.W.; Wade, M.M.; Holman, S.C.; Champlin, F.R. Status of methods for assessing bacterial cell surface charge properties based on Zeta potential measurements. J. Microbiol. Methods 2001, 43, 153-164. [CrossRef]

35. Houston, D.M.; Bugert, J.J.; Denyer, S.P.; Heard, C.M. Anti-inflammatory activity of Punica granatum L. (pomegranate) rind extracts applied topically to ex vivo skin. Eur. J. Pharm. Biopharm. 2017, 112, 30-37. [CrossRef] 
36. Celiksoy, V.; Moses, R.L.; Sloan, A.J.; Moseley, R.; Heard, C.M. Evaluation of the in vitro oral wound healing effects of pomegranate (Punica granatum) rind extract and punicalagin, in combination with Zn (II). Biomolecules 2020, 10, 1234. [CrossRef]

37. Sharquie, K.E.; Al-Mashhadani, S.A.; Salman, H.A. Topical 10\% zinc sulfate solution for treatment of melasma. Dermatol. Surg. 2008, 34, 1346-1349. [CrossRef] [PubMed]

38. Sharquie, K.E.; Al-Mashhadani, S.A.; Noaimi, A.A.; Hasan, A.A. Topical zinc sulphate (25\%) solution: A new therapy for actinic keratosis. J. Cutan. Aesthet. Surg. 2012, 5, 53-56. [CrossRef] [PubMed]

39. Gupta, M.; Mahajan, V.K.; Mehta, K.S.; Chauhan, P.S. Zinc therapy in dermatology: A review. Dermatol. Res. Pract. 2014, 2014, 709152. [CrossRef] [PubMed] 